\title{
FOLDING OF A LAYERED VISCOELASTIC MEDIUM DERIVED FROM AN EXACT STABILITY THEORY OF A CONTINUUM UNDER INITIAL STRESS*
}

\author{
$\mathrm{Br}$ \\ M. A. BIOT \\ Shell Development Company, New York
}

\begin{abstract}
A layer of viscoelastic material embedded in an infinite medium also viscoelastic tends to fold through instability when the system is compressed in a direction parallel with the layer. This phenomenon which was treated previously by using an approximate plate theory, [1, 2], is analyzed here by using an exact theory for the deformation of a continuum under prestress $[3,4,5,6]$. The effect of the compressive prestress in the embedding medium is taken into account, and it is found that although it is not always negligible, it tends to be very small under conditions where the instability of the layer is strong. The same conclusion holds for the error involved in the use of a plate theory for the layer instead of the exact equations for a prestressed continuum. In the course of the analysis we have also treated the problem of a semi-infinite viscoelastic half space subject to a uniform internal compression parallel with the boundary and a surface load normal to this boundary. The compressive load produces an increase of the surface deflection under the normal load. This effect appears through an amplification factor which is evaluated numerically for the particular example of an elastic body. It is shown that under certain conditions the free surface of the compressed semiinfinite medium may become unstable and will tend to wrinkle. This is suggested as a probable explanation for the wrinkles which appear on the surface of a body subjected to a plastic compression.
\end{abstract}

1. Introduction. We consider a layer of viscoelastic material surrounded by an unbounded medium also viscoelastic, the whole system being subject to compressive stresses parallel with the direction of the layer. Such stresses may be set up for instance if the whole system is subject to a uniform compressive strain parallel with the layer.

As another example we have the case where a compressive stress in the layer alone will be produced if a swelling of the layer occurs while longitudinal expansion is prevented. The question which we are concerned with here is the stability of the layer under such a system of prestress and the prediction of the deformation of this sytem. We have already analyzed this problem by introducing certain approximations [1, 2]. In particular we have assumed that the layer behaves like a plate, i.e., that it obeys equations of flexure of the type used in strength of materials theory and generalized to apply to viscoelastic media. Another assumption introduced in this previous work is that the prestress in the surrounding medium has a negligible effect.

Our purpose is to check the accuracy of the previous simplified theories by solving the problem from the exact equations for small deformations of a continuum under prestress. General equations for incremental deformations of a body under prestress were derived by this writer some years ago in a series of publications $[3,4,5,6]$. While the theory was developed in the particular context of elastic continua, the equations

\footnotetext{
*Received June 26, 1958.
} 
are applicable to any type of incremental deformations of elastic or anelastic bodies. This is due to the fact that in the development of the theory the physics is separated from the geometry of the problem, and the incremental stress strain relations are left arbitrary. For the reader's convenience, we have rederived the equations in Sec. 2 for the particular case of plane strain. In Sec. 3 these equations are first applied to the evaluation of the surface deflection under a normal load for a semi-infinite viscoelastic medium, which is at the same time subject to a compression in a direction parallel with the boundary.

The relations between the incremental stresses and strains are assumed to be the same as those derived by the present writer from thermodynamics [7]. The nature of the approximation which may be involved in this assumption is discussed in a previous publication [2]. It is found that the lateral compression produces an amplification of the surface deflection and its magnitude is evaluated. The possibility of an instability of the free surface under a lateral compression is analyzed in Sec. 4 . It is pointed out that although the magnitude of this instability is in general quite small, the effect may be large for incremental deformations in the plastic range where the so-called tangent modulus is the significant parameter. The instability should manifest itself in the form of a wrinkling of the surface, a phenomenon which is actually observed on the surface of a compressed solid in the plastic range.

In Sec. 5 a complete analysis is given for the folding of a layer of uniform thickness embedded in a medium of infinite extent. We have assumed that there is no friction at the interface of the layer and the medium. This affords a direct comparison with the results obtained in [1] and [2] by an approximate theory and with the same assumption of perfect interfacial slip.* Results of the previous section are used in order to evaluate the influence of the compressive stress in the surrounding medium. It is found that in general this compression may be neglected, as it does not affect substantially the wave length of the folding. The case where the layer and the surrounding medium are both incompressible viscous fluids is investigated in detail.

The rate of growth of the amplitude of folding as a function of the wave length has been derived. The dominant wave length-i.e., that which exhibits the maximum rate of amplitude growth - is evaluated as a function of the viscosity ratio of the two media. This dominant wave length is independent of the load. Results are compared with the results of the approximate theory of [1]. It is found that this approximate theory agrees very well with the present results when the layer viscosity is larger than about seventy times the viscosity of the surrounding medium. This is also the condition for the magnitude of the instability to become significant so that the folding amplitude increases at a rate fast enough to be observable in practice.

2. The theory of deformation of a prestressed solid. We shall first recall briefly the theory of incremental stress and deformation of a solid under initial stress. We assume the incremental deformation to be small so that the theory is linear. Such a theory was developed by the writer $[3,4,5,6]$ for the case of an elastic body. However, it is independent of the physical nature of the body and is therefore applicable to viscoelastic media. In order to simplify the presentation, we shall limit ourselves here to a two dimensional deformation.

We are dealing with the problem of a continuum undergoing small deformation

*An evaluation of the effect of interfacial adherence has been given in [2]. 
from an initial state which is not one of zero stress. The initial stress field is defined by components $S_{11}, S_{12}=S_{21}, S_{22}$, referred to $x, y$ axes. This being a two dimensional case, we assume that the other components of the initial stress field vanish or do not appear in the equations. The condition of equilibrium of this stress field is

$$
\begin{aligned}
& \frac{\partial S_{11}}{\partial x}+\frac{\partial S_{12}}{\partial y}+\rho X=0, \\
& \frac{\partial S_{21}}{\partial x}+\frac{\partial S_{22}}{\partial y}+\rho Y=0,
\end{aligned}
$$

with a mass density $\rho$ and a body force $X, Y$ per unit mass. The problem is to formulate the laws of deformation of this medium where small incremental stresses and strains are superimposed on the initial state.

In treating this problem we have departed from the traditional viewpoint of the mathematician and defined the incremental tensors in a different way. Let us first consider the strain. A point $P$ of initial coordinates $x, y$ in the prestressed state is displaced to $P^{\prime}$ of coordinates

$$
\begin{aligned}
& \xi=x+u, \\
& \eta=y+v .
\end{aligned}
$$

In this transformation the material around $P$ undergoes a rotation

$$
\omega=\frac{1}{2}\left(\frac{\partial v}{\partial x}-\frac{\partial u}{\partial y}\right)
$$

and a deformation

$$
e_{x x}=\frac{\partial u}{\partial x} \quad e_{y y}=\frac{\partial v}{\partial y} \quad e_{x y}=\frac{1}{2}\left(\frac{\partial v}{\partial x}+\frac{\partial u}{\partial y}\right) .
$$

In order to deal with the problem of relating stress to strain, it is important to consider the phenomenon from the view point of an observer rotating with the material through an angle $\omega$. If we denote by $E_{i j}$ the strain components referred to rotated axes, then it is clear that to the first order these are the same as $e_{i j}$ defined above,

$$
E_{i j}=e_{i i} .
$$

This is not true, however, for the incremental stress. If we refer the stress to axes rotating with the material, the total stress acting on the element is

$$
\left\{\begin{array}{ll}
S_{11}+s_{11} & S_{12}+s_{12} \\
S_{21}+s_{21} & S_{22}+s_{22}
\end{array}\right\} .
$$

The incremental stress $s_{i i}$ is referred to rotated axes and depends only on the strain $e_{i j}$. The nature of this relation is a physical problem which we shall consider in the next section. We shall deal here only with the geometrical aspects of the problem and established equations for the incremental stress field $s_{i i}$ which express the condition of equilibrium of the field. This is done by first referring the stress field to the unrotated initial directions $x, y$. These stress components are to the first order 


$$
\begin{aligned}
\sigma_{x x} & =S_{11}+s_{11}-2 S_{12} \omega, \\
\sigma_{y y} & =S_{22}+s_{22}+2 S_{12} \omega, \\
\sigma_{x y} & =S_{12}+s_{12}+\left(S_{11}-S_{22}\right) \omega .
\end{aligned}
$$

Note that in the deformation this stress field is the one at point $\xi=x+u$ and $\eta=y+\sigma$. Also that the mass density has now become $\rho$ ' by the incremental volume change.* The equations of equilibrium of the field $\sigma_{i j}$ are

$$
\begin{aligned}
& \frac{\partial \sigma_{x x}}{\partial \xi}+\frac{\partial \sigma_{x y}}{\partial \eta}+\rho^{\prime} X=0, \\
& \frac{\partial \sigma_{x y}}{\partial \xi}+\frac{\partial \sigma_{y y}}{\partial \eta}+\rho^{\prime} Y=0 .
\end{aligned}
$$

We now express these equations by means of the original coordinates $x, y$. Using the relations

$$
\begin{aligned}
& d \xi=\left(1+\frac{\partial u}{\partial x}\right) d x+\frac{\partial u}{\partial y} d y \\
& d \eta=\frac{\partial v}{\partial x} d x+\left(1+\frac{\partial v}{\partial y}\right) d y
\end{aligned}
$$

and solving for $d x, d y$, we find the partial derivatives of $x, y$ with respect to $\xi, \eta$, e.g.

$$
\frac{\partial x}{\partial \xi}=\frac{1}{D}\left(1+\frac{\partial v}{\partial y}\right) \text { etc. }
$$

$D$ is the Jacobian, i.e., the determinant of the transformation (2.9). With these expressions we may write

$$
\frac{\partial \sigma_{x x}}{\partial \xi}=\frac{\partial \sigma_{x x}}{\partial x} \frac{\partial x}{\partial \xi}+\frac{\partial \sigma_{x x}}{\partial y} \frac{\partial y}{\partial \xi}=\frac{1}{D} \frac{\partial \sigma_{x x}}{\partial x}\left(1+\frac{\partial v}{\partial y}\right)-\frac{1}{D} \frac{\partial \sigma_{x x}}{\partial y} \frac{\partial v}{\partial x} .
$$

Conservation of mass requires

$$
D \rho^{\prime}=\rho .
$$

The equilibrium equations (2.8) may then be expressed in terms of $x$ and $y$. Substituting the values (2.7) for $\sigma_{i j}$ retaining only first order terms, gives

$$
\begin{aligned}
\frac{\partial s_{11}}{\partial x}+\frac{\partial s_{12}}{\partial y}-2 \frac{\partial}{\partial x}\left(S_{12} \omega\right)+ & \frac{\partial}{\partial y}\left[\left(S_{11}-S_{22}\right) \omega\right] \\
& +\frac{\partial S_{11}}{\partial x} \frac{\partial v}{\partial y}-\frac{\partial S_{11}}{\partial y} \frac{\partial v}{\partial x}-\frac{\partial S_{12}}{\partial x} \frac{\partial u}{\partial y}+\frac{\partial S_{12}}{\partial y} \frac{\partial u}{\partial x}=0 .
\end{aligned}
$$

Another equation is obtained by permutation of the symbols and changing $\omega$ in $-\omega$. These equations are further simplified by introducing the following identities

$$
\begin{aligned}
& \frac{\partial v}{\partial x}=e_{x y}+\omega \\
& \frac{\partial u}{\partial y}=e_{x y}-\omega
\end{aligned}
$$

${ }^{*}$ For simplicity we assume here that the body force is independent of the coordinates. The more general case is considered in [4]. 
and by taking into account the equilibrium condition (2.1) for the initial stress field. Equations (2.13) are then written

$$
\begin{aligned}
\frac{\partial s_{11}}{\partial x}+\frac{\partial s_{12}}{\partial y}+\rho Y \omega-2 S_{12} \frac{\partial \omega}{\partial x} & +\left(S_{11}-S_{22}\right) \frac{\partial \omega}{\partial y} \\
& +\frac{\partial S_{11}}{\partial x} e_{y y}-\left(\frac{\partial S_{11}}{\partial y}+\frac{\partial S_{12}}{\partial x}\right) e_{x y}+\frac{\partial S_{12}}{\partial y} e_{x x}=0 .
\end{aligned}
$$

The other equation is also obtained by permutation of the variables and changing $\omega$ in $-\omega$. If the initial field is uniform, the terms written on the second line disappear and the equations become

$$
\begin{aligned}
& \frac{\partial s_{11}}{\partial x}+\frac{\partial s_{12}}{\partial y}+\rho Y \omega-2 S_{12} \frac{\partial \omega}{\partial x}+\left(S_{11}-S_{22}\right) \frac{\partial \omega}{\partial y}=0, \\
& \frac{\partial s_{12}}{\partial x}+\frac{\partial s_{22}}{\partial y}-\rho X \omega+2 S_{12} \frac{\partial \omega}{\partial y}+\left(S_{11}-S_{22}\right) \frac{\partial \omega}{\partial x}=0 .
\end{aligned}
$$

It is interesting to see that in the absence of a body force the only additional terms due to the pre-stress depend on the total initial shear and disappear if the initial stress is hydrostatic.

The boundary conditions are found from the stresses $\sigma_{i j}$. The force acting on an arc element $d \xi, d \eta$, after deformation is

$$
\begin{aligned}
& d F_{x}=\sigma_{x x} d \eta-\sigma_{x y} d \xi, \\
& d F_{\nu}=\sigma_{x y} d \eta-\sigma_{y y} d \xi .
\end{aligned}
$$

The force $F$ is acting on matter lying to the left side of the arc element $d \xi, d \eta$ in a counterclockwise coordinate system and to the right in a clockwise system. Substituting the values (2.7) for the stresses, expressions (2.9) for $d \xi, d \eta$ and retaining only first order terms, we find

$$
\begin{aligned}
& d F_{x}=-\left[s_{12}+S_{12}-S_{22} \omega+S_{12} e_{x x}-S_{11} e_{x y}\right] d x \\
& +\left[s_{11}+S_{11}-S_{12} \omega+S_{11} e_{y y}-S_{12} e_{x y}\right] d y, \\
& d F_{y}=-\left[s_{22}+S_{22}+S_{12} \omega+S_{22} e_{x x}-S_{12} e_{x y}\right] d x \\
& +\left[s_{12}+S_{12}+S_{11} \omega+S_{12} e_{y y}-S_{22} e_{x y}\right] d y .
\end{aligned}
$$

This gives the force acting on an element of arc initially of components $d x, d y$, at point $x, y$.

3. The viscoelastic half space under combined surface loading and lateral compression. We shall apply the previous equations to the problem of determining the deflection of the surface of a viscoelastic half space under the action of a load normal to the surface and a condition of prestress which is a compression in a direction parallel to the surface (Fig. 1).

The surface is along the $x$-axis and the $y$-axis is directed inward. The initial stress system is assumed to be

$$
S_{22}=S_{12}=0, \quad S_{11}=-P,
$$

i.e., a uniform compression along the $x$-direction. We assume the body force to be zero. Equations (2.16) become 


$$
\begin{aligned}
& \frac{\partial s_{11}}{\partial x}+\frac{\partial s_{12}}{\partial y}-P \frac{\partial \omega}{\partial y}=0 \\
& \frac{\partial s_{12}}{\partial x}+\frac{\partial s_{22}}{\partial y}-P \frac{\partial \omega}{\partial x}=0
\end{aligned}
$$

The next step is now to introduce a relation between the incremental stresses $s_{i j}$ and the incremental strain $e_{i j}$. Such relations have not yet been developed in the most general case for a pre-stressed material, and some difficulty arises in this connection. We have shown [4] that for an elastic body under prestress, the elastic constants cannot be the same as in the unstressed condition unless the prestress system is hydrostatic. We therefore point out that, in general, it must be considered an approximation to use -as we shall do here-the same stress-strain relations for the prestressed and the initially stress-free medium. We have discussed this point more extensively in [2].

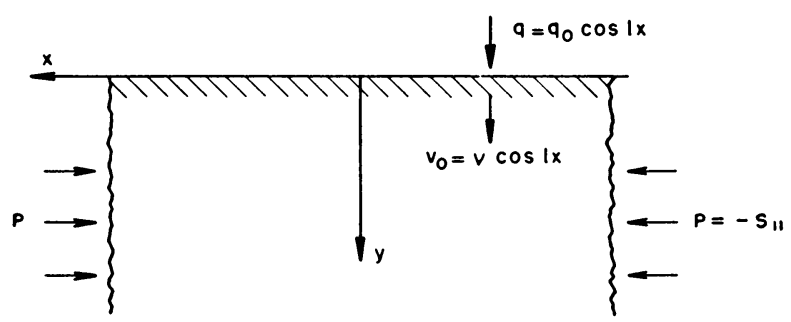

Fig. 1. Viscoelastic half space under combined surface loads ąnd lateral compression.

Another point arises if the body is viscous for slow deformations. Then the initial state is one of steady strain rate, and there are additional incremental strains which are not due to the incremental stresses $s_{i j}$ alone.

In the applications below we shall, therefore, consider the total displacement field as $u_{i}+u_{i}^{*}$ where $u_{i}^{*}$ is the displacement associated with the steady strain rate due to the initial stress $S_{i j}$. The term $u_{i}$ then represents the departure of the displacement from the initial uniform strain rate. The incremental strain and rotation appearing in equations (2.15), (2.16) and (2.18) should then be written as $e_{i j}+e_{i j}^{*}$ and $\omega+\omega^{*}$ where $e_{i j}^{*}$ and $\omega^{*}$ correspond to the initial steady state.

In the applications below, the components $e_{i j}^{*}$ and $\omega^{*}$ drop out of the equations and do not appear in the formulation of the problem. We may, therefore, disregard the term $u_{i}^{*}$ in the present treatment. In general, of course, since $e_{i j}^{*}$ and $\omega^{*}$ are linear functions of time, they will disappear from the equations by introducing second time derivatives of all variables.

The operational stress-strain relations for an initially stress-free medium were established in [7] and found to be

$$
s_{i j}=2 \bar{Q} e_{i j}+\delta_{i j} \bar{R} e
$$

with the unit matrix $\delta_{i j}$ and $e=e_{x x}+e_{y y}$.

The operators are

$$
\begin{aligned}
\bar{Q} & =p \int_{0}^{\infty} \frac{Q(r)}{p+r} \gamma(r) d r+Q+Q^{\prime} p \\
\bar{R} & =p \int_{0}^{\infty} \frac{R(r)}{p+r} \gamma(r) d r+R+R^{\prime} p
\end{aligned}
$$


where $p$ designates the time differential operator

$$
p=\frac{d}{d t} \text {. }
$$

By a well-known property of such operators, the relations are also valid if $p$ is a real or a complex quantity, provided all variables contain the exponential factor $e^{p t}$.

Substituting expressions (3.3) for the stress into the equations (3.2), we find

$$
\begin{gathered}
\bar{Q} \nabla^{2} u+[\bar{Q}+\bar{R}] \frac{\partial e}{\partial x}-P \frac{\partial \omega}{\partial y}=0, \\
\bar{Q} \nabla^{2} v+[\bar{Q}+\bar{R}] \frac{\partial e}{\partial y}-P \frac{\partial \omega}{\partial x}=0 .
\end{gathered}
$$

It can be verified that solutions of these equations with a sinusoidal factor along $x$ are

$$
\begin{aligned}
& u=-\sin l x\left[A e^{-l y}+C k(1-\beta) e^{-l k \nu}\right], \\
& v=-\cos l x\left[A e^{-l y}+C\left(1-\beta k^{2}\right) e^{-l k y}\right] .
\end{aligned}
$$

These solutions are analogous to those developed in $[3,6]$ for the purely elastic case. In these expressions $A$ and $C$ are unknown operators, and we have put

$$
2 \beta=\frac{2 \bar{Q}+P}{2 \bar{Q}+\bar{R}}, \quad k=\left(\frac{1-\zeta}{1+\zeta}\right)^{1 / 2}, \quad \zeta=\frac{P}{2 \bar{Q}} .
$$

If $k$ is chosen positive the solutions (3.8) represent deformations confined to a region near the surface. The same is true if $k$ is complex and chosen such that its real part is positive.

Consider now the case where a normal load of sinusoidal distribution is applied to the surface $(y=0)$. We wish to determine the deflection at the surface due to the simultaneous action of the surface load and the compressive preload $P$ in the material. The surface load will be represented as

$$
\frac{d F_{y}}{d x}=q=q_{0} \cos l x .
$$

It is acting downward in the $y$ direction as indicated in Fig. 1. The tangential component of the boundary force vanishes

$$
d F_{x}=0 .
$$

Introducing expressions (2.18) into the relations (3.10) (3.11), we obtain the boundary conditions

$$
\begin{aligned}
& e_{x y}=0, \\
& s_{22}=-q_{0} \cos l_{x}=2 \bar{Q} e_{y y}+\bar{R} e .
\end{aligned}
$$

Substituting in these conditions the values $u$ and $v$ from expressions (3.8) and putting $y=0$ yields

$$
\begin{gathered}
A+\frac{C}{2}\left(1+k^{2}-2 \beta k^{2}\right)=0 \\
A+C k\left(1-\beta k^{2}\right)+\frac{C \bar{R}}{2 \bar{Q}} k \beta\left(1-k^{2}\right)=-\frac{q}{2 \bar{Q} l} .
\end{gathered}
$$


The conditions determine the operators $A$ and $C$. The deflection of the surface is also sinusoidal and represented by

$$
v_{0}=V \cos l x .
$$

The ratio of the load $q$ to the deflection $v_{0}$ at the surface $y=0$ is then given by

$$
\frac{q_{0}}{V l}=\frac{q}{v_{0} l}=\frac{1}{2} B\left[\frac{k(1+\zeta)^{2}-1}{\zeta}-\alpha \zeta\right]
$$

with the notation

$$
\begin{aligned}
B & =\frac{4 \bar{Q}(\bar{Q}+\bar{R})}{2 \bar{Q}+\bar{R}}, \\
\alpha & =\frac{\bar{Q}}{\bar{Q}+\bar{R}} .
\end{aligned}
$$

The effect of the compressive load $P$ arises through the bracketed factor in Eq. (3.15). This factor is

$$
\varphi=\frac{1}{\zeta}\left[k(1+\zeta)^{2}-1\right]-\alpha \zeta .
$$

The deflection under a load $q_{0}$ is

$$
V=\frac{2}{l B \varphi} q_{0} .
$$

If a constant sinusoidally distributed load is suddenly applied at time $t=0$ with a maximum value unity,

$$
q_{0}=1(t)= \begin{cases}0 & t<0 \\ 1 & t>0\end{cases}
$$

it may be represented by the complex integral

$$
q_{0}=1(t)=\frac{1}{2 \pi i} \int_{C-i \infty}^{c+i \infty} \frac{e^{p t}}{p} d p .
$$

Hence the deflection under this load is

$$
V(t)=\frac{1}{i l \pi} \int_{C-i \infty}^{c+i \infty} \frac{e^{p t}}{p B \varphi} d p .
$$

In this expression $\bar{B}$ and $\varphi$ are functions of $p$.

The effect of the lateral compression $P$ appears through the factor $\varphi$. If this lateral compression is zero, then

$$
\zeta=0, \quad \varphi=1
$$

and the deflection is

$$
V(t)=\frac{1}{i l \pi} \int_{C-i \infty}^{c+i \infty} \frac{e^{p t}}{p B} d p .
$$

In order to give an idea of the magnitudes involved, let us consider first the case of a 
purely elastic incompressible material. Incompressibility corresponds to

$$
\bar{R}=\infty \quad \text { or } \alpha=0 .
$$

With a shear modulus $G$, we put

$$
\bar{Q}=G
$$

Hence

$$
\varphi=\frac{k(1+\zeta)^{2}-1}{\zeta}
$$

with

$$
\zeta=P / 2 G .
$$

The deflection is amplified by the lateral compression $P$, and the amplification factor is $1 / \varphi$. Values of this amplification factor as a function of $\zeta$ are given in Table 1 .

TABLE 1.

\begin{tabular}{c|c}
\hline \hline$\zeta$ & $1 / \varphi$ \\
\hline 0.50 & 1.67 \\
\hline 0.60 & 2.14 \\
\hline 0.70 & 3.29 \\
\hline 0.80 & 10.00 \\
\hline 0.85 & $\infty$ \\
\hline
\end{tabular}

We see that when $\zeta=0.85$ i.e. for $P=1.7 G$ the amplification factor is infinite and the surface becomes unstable. We also notice that the amplification factor becomes appreciable only for values $\zeta>0.5$; hence, for compressive loads $P$ larger than $G$ these loads are of the order of the elastic modulus. In a material of constant modulus, they can only occur for loads corresponding to a very large compressive strain. However, we must remember that the present theory is for incremental deformations. Hence, the modulus $G$ appearing in the above expression is actually the so-called tangent modulus. In many cases where plastic deformations are involved, this modulus decreases with the load, and the above values may well fall within observable ranges.

In regard to the significance of the amplification factor, it should be remarked that it is the same for all wave lengths. Hence, the deflection of the surface under an arbitrary load distribution may be derived from the deflection under the same surface load and zero compression $(P=0)$ by simply multiplying the latter by the amplification factor. The compressive load introduces no distortion of the deflection but simply a change of magnitude.

It can easily be seen that the amplification effect of the compressive load on the surface deflection of a half space also applies when the initial deflection is not due to a surface loading but due to irregularities and waviness. This departure from perfect 
smoothness is amplified without distortion by the compressive loads. This suggests that under high compressive loads, surfaces which appear to be smooth initially will tend to wrinkle. Since the amplification factor is dependent only on the tangent modulus, this mechanism suggests itself particularly as the explanation for the wrinkling which appears on surfaces of materials compressed in the plastic range.

4. Instability of the free surface of a half space under compression. Let us now examine the condition of instability of the free surface of a half space under compression. This corresponds to the existence of solutions different from zero for the deflection $V$ while the surface load $q_{0}$ is zero. Equation (3.18) may be written

$$
q_{0}=\frac{1}{2} B \varphi V l \text {. }
$$

The condition $q_{0}=0$ with $V \neq 0$ amounts to

$$
\varphi=\frac{1}{\zeta}\left[k(1+\zeta)^{2}-1\right]-\alpha \zeta=0
$$

which may also be written

$$
\frac{k(1+\zeta)^{2}}{\zeta}=\frac{1+\alpha \zeta^{2}}{\zeta}
$$

or

$$
2-2 \zeta^{2}-\zeta^{3}=2 \alpha \zeta+\alpha^{2} \zeta^{3}
$$

Let us first examine the elastic case. The operators $\bar{Q}$ and $\bar{R}$ are replaced by the Lamé constants

$$
\bar{Q}=G \quad \bar{R}=\lambda .
$$

Poisson's ratio being designated by $\nu$ we find

$$
\alpha=\frac{G}{\lambda+G}=1-2 \nu .
$$

Also

$$
\zeta=P / 2 G .
$$

The critical value of $\zeta$ is the real root of Eq. (4.4). This root depends on Poisson's ratio only and its value is shown in Table 2 .

TABLE 2

\begin{tabular}{l|l}
\hline \hline$\nu$ & $\zeta$ \\
\hline 0 & .55 \\
$\frac{1}{4}$ & .68 \\
$\frac{1}{2}$ & .84 \\
\hline
\end{tabular}

The instability occurs at values of the compressive load $P$ comparable with the shear modulus $G$. However, as already pointed out above, since the latter is the tangent modulus, its value may be low enough in the plastic range for the surface instability to occur within the practical range of $P$. 
As an example of a viscous material, let us now consider the case of a viscous incompressible fluid. Some clarification is needed here as to what is meant by instability. We assume that the half space is initially under the influence of a compressive load $P$ parallel with the free surface and that the fluid is initially in a steady state of uniform rate of deformation under this load. All velocities and strain rates are initially constant in time and space. The stability of the free surface under those conditions is determined by considering the possible exponential growth of a disturbance starting from a certain instantaneous configuration considered as the initial state.* The possibility of such instability is easily determined from the above analysis. For an incompressible fluid, the operators become

$$
\bar{Q}=\mu p, \quad \bar{R}=\infty,
$$

with $\mu$ the viscosity coefficient. Since the condition $\bar{R}=\infty$ implies $\alpha=0$, Eq. (4.4) becomes

$$
2-2 \zeta^{2}-\zeta^{3}=0
$$

with

$$
\zeta=\frac{P}{2 \bar{Q}}=\frac{P}{2 \mu p} .
$$

Equation (4.9) is a cubic in the unknown $p$. For values of $p$ which are roots of this equation, there exist disturbances proportional to the factor $e^{p t}$. Instability corresponds to roots which are real and positive or complex with a positive real part. The three roots $p$ of $(4.9)$ are

$$
\begin{aligned}
& p_{1}=1.192 \frac{P}{2 \mu}, \\
& p_{2}=(-0.596+0.255 i) \frac{P}{2 \mu}, \\
& p_{3}=(-0.596-0.255 i) \frac{P}{2 \mu} .
\end{aligned}
$$

When substituted in Eq. (4.2), i.e. $\varphi=0$, the complex roots do not furnish a solution if we choose $k$ to have a positive real part. Hence they do not correspond to the physical problem.

The root $p_{1}$ corresponds to instability. In order to appreciate the magnitude of this instability, let us consider a time $t_{1}$ during which the undisturbed uniform flow produces a $25 \%$ compressive strain. The rate of strain in the $x$ direction under the load $P$ is

$$
\dot{e}_{x x}=-\frac{0.25}{t_{1}}=-\frac{P}{4 \mu} \text {. }
$$

Hence,

$$
t_{1}=\frac{\mu}{P}, \quad p_{1} t_{1}=0.596
$$

\footnotetext{
*In dealing with a viscous fluid whose initial state of stress gives rise to a steady strain rate, we must refer to the remark Sec. 3 by which it is justified to consider not the actual incremental deformation, but the departure of the deformation from the steady state itself.
} 
This means that in the time interval $t_{1}$ during which the material is squeezed by $25 \%$, any disturbance of the free surface from a perfect plane is amplified by a factor $e^{\boldsymbol{p}_{1} t_{2}}=$ 1.81. This instability is of such a mild form that it will be very difficult to observe in practice.*

A similar procedure may be used for any kind of viscoelastic half space by solving Eq. (4.4) as an equation for $p$ with the operators $\bar{Q}$ and $\bar{R}$ as appropriate functions of the unknown $p$.

5. Characteristic equation for the stability of the embedded layer. We consider a layer of thickness $h$ embedded in an infinite medium. The layer and the surrounding medium have different viscoelastic properties. The layer is subject to an initial compression $P$ and the surrounding medium to a compression $P_{1}$ (Fig. 2). This initial state

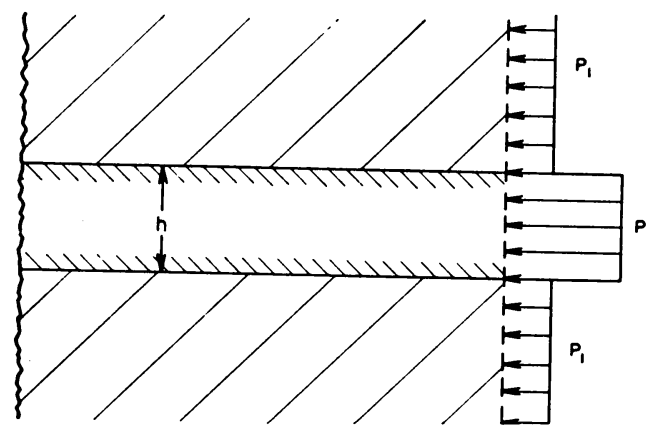

FIG. 2. Layer embedded in infinite medium under lateral compression.

of stress will depend on the type of initial deformation imposed on the system and on the viscoelastic parameters of the two media. For example, if the two media are incompressible viscous fluids and if the system is squeezed uniformly in a direction parallel with the layer, the compressive stresses are in the ratio

$$
\frac{P}{P_{1}}=\frac{\mu}{\mu_{1}},
$$

where $\mu$ is the viscosity coefficient of the layer and $\mu_{1}$ that of the surrounding medium. The stress could also be due to other causes, as, for instance, an increase of volume of the layer while longitudinal elongation is prevented. In this case the stress $P_{1}$ in the surrounding medium can be zero.

In order to simplify the formulation, we shall assume that the interface between the layer and the medium is perfectly lubricated so that there are no tangential stresses at the interface. A previously developed approximate theory for the case of perfect and imperfect adherence [2] indicates that this assumption does not modify the result substantially. The properties of the layer are represented by the operators $\bar{Q}$ and $\bar{R}$ and that of the surrounding medium by $\bar{Q}_{1}$ and $\cdot \bar{R}_{1}$. We consider the layer separately and locate the $x$-axis in the plane equidistant from the two faces (Fig. 3). The $y$-axis is normal to the layer. Flexure of the layer is represented by antisymmetric solutions of Eqs.

\footnotetext{
*Since the theory is only valid for small deformations, its application to the case of a large compressive strain of 25 per cent will, of course, give only an indication of the orders of magnitude of the amplification effect.
} 


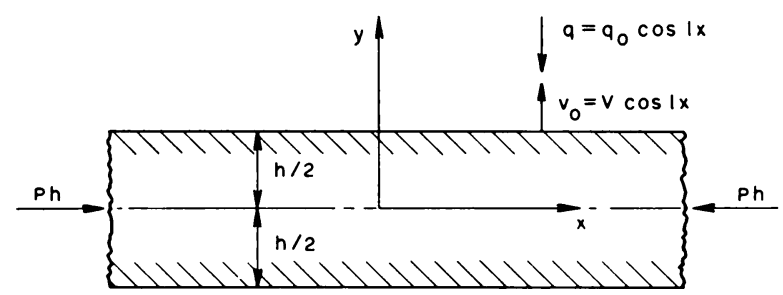

FIG. 3. Forces acting on the layer.

(3.7). They are obtained by adding two solutions of the type (3.8) by using both positive and negative exponents. We find

$$
\begin{aligned}
& u=-\sin l x[A \sinh l y+C k(1-\beta) \sinh l k y], \\
& v=\cos l x\left[A \cosh l y+C\left(1-\beta k^{2}\right) \cosh l k y\right],
\end{aligned}
$$

where $\beta$ and $k$ are defined by (3.9).

This also coincides with the solution obtained previously for the purely elastic case [3]. The normal force applied to the layer at the boundary $y=h / 2$ and due to the surrounding medium is

$$
q=q_{0} \cos l x .
$$

This load is taken positive in the negative $y$ direction, and the tangential force vanishes owing to the condition of perfect slip at the interface. Because of symmetry, boundary conditions imposed at one interface are automatically satisfied at the other. We shall consider the interface at $y=h / 2$. Applying (2.18) we find the boundary conditions

$$
\begin{aligned}
& \frac{d F_{x}}{d x}=-s_{12}-P e_{x y}=0 \quad y=\frac{h}{2} \\
& \frac{d F_{y}}{d x}=-s_{22}=-q_{0} \cos l x .
\end{aligned}
$$

From expressions (3.3) for $s_{12}$ and $s_{22}$ in terms of the strains we find at $y=h / 2$,

$$
\begin{gathered}
e_{x y}=0, \\
2 \bar{Q} e_{y y}+\bar{R}\left(e_{x x}+e_{y y}\right)=-q_{0} \cos l x .
\end{gathered}
$$

Substituting the expressions (5.2) for $u$ and $v$ yields two equations for the operators $A$ and $C$ in terms of $q_{0}$. We must also consider the boundary value of the normal displacement $v$. This is expressed by substituting $y=h / 2$ in the second equation (5.2). We find

$$
v=V \cos l x=\left[A \cosh \frac{l h}{2}+C\left(1-\beta k^{2}\right) \cosh \frac{l k h}{2}\right] \cos l x .
$$

The normal deflection at the boundary $y=h / 2$ is $V \cos l x$. By substituting in (5.6) the expressions for $A$ and $C$ determined from the two equations (5.5), we find a relation between the load $q_{0}$ and the interface deflection $V$. This relation may be written

$$
\frac{2 q_{0}}{l V B}=\frac{1}{\zeta}\left(1+\alpha \zeta^{2}\right) \tanh \gamma-\frac{(1+\zeta)^{2}}{\zeta} k \tanh k \gamma,
$$


where

$$
\gamma=\frac{1}{2} l h, \quad \zeta=\frac{P}{2 \bar{Q}},
$$

and $B$ and $\alpha$ are operators depending solely on the viscoelastic properties of the layer

$$
\begin{aligned}
\alpha & =\frac{\bar{Q}}{\bar{Q}+\bar{R}}, \\
B & =\frac{4 \bar{Q}(\bar{Q}+\bar{R})}{2 \bar{Q}+\bar{R}} .
\end{aligned}
$$

At this point we still have to match boundary conditions for the layer and the half space representing the embedding medium. This is readily done if we consider the ratio of the load $q_{0}$ to deflection $V$ for the surface of the half space which was obtained in Sec. 3 and given by expression

$$
\frac{2 q_{0}}{l V}=B_{1} \varphi_{1}
$$

where

$$
\begin{gathered}
B_{1}=\frac{4 \bar{Q}_{1}\left(\bar{Q}_{1}+\bar{R}_{1}\right)}{2 \bar{Q}_{1}+\bar{R}_{1}}, \\
\varphi_{1}=\frac{2-2 \zeta_{1}^{2}-\zeta_{1}^{3}}{k_{1}\left(1+\zeta_{1}\right)^{2}+1}-\alpha_{1} \zeta_{1}, \\
\zeta_{1}=\frac{P_{1}}{2 \bar{Q}_{1}} \quad k_{1}=\left(\frac{1-\zeta_{1}}{1+\zeta_{1}}\right)^{1 / 2} \quad \alpha_{1}=\frac{\bar{Q}_{1}}{\bar{Q}_{1}+\bar{R}_{1}} .
\end{gathered}
$$

The quantities $B_{1}$ and $\alpha_{1}$ depend only on the viscoelastic properties of the surrounding medium, while the others include the value of the prestress $P_{1}$ in this medium. This prestress is contained in the expression $\varphi_{1}$ and, as was pointed out above, we may consider $1 / \varphi_{1}$ as an amplification factor for the deflection at the surface of a half space. The value of the amplification factor is unity when the prestress is zero $\left(P_{1}=\zeta_{1}=0\right)$. Since the load $q_{0}$ and the normal deflection $V$ are the same for both the layer and the medium at the interface matching of the boundary, conditions amount to equating the values of $q_{0} / l V$ appearing on the left side of the two equations (5.7) and (5.9). This yields the characteristic equation

$$
\frac{B_{1}}{B} \varphi_{1}=\frac{1}{\zeta}\left(1+\alpha \zeta^{2}\right) \tanh \gamma-\frac{(1+\zeta)^{2}}{\zeta} k \tanh k \gamma
$$

It is true that the values of $q_{0}$ and $V$ for the layer and the medium are not those of the same location of the interface because of the relative slip of the two media. However, the error is of the second order and is irrelevant in a first order theory.

The parameters of the surrounding medium are contained solely in $B_{1} \varphi_{1}$ on the left hand side. The significance of this characteristic equation lies in the fact that for given materials and a given prestress field $P$ and $P_{1}$, it constitutes a relation between the nondimensional wave number $\gamma=\frac{1}{2} l h$ of the folding and the value of $p$. This value of $p$ is the coefficient in the time factor $e^{p t}$ which multiplies the amplitude of the folding for 
any particular wave number $\gamma$ initially present as a disturbance in the layer. In a system with random initial disturbance, those wave lengths will appear for which $p$ has the largest positive value. We shall disregard the possibility that there are complex values for $p$ as encountered above in the case of the viscoelastic half space and assume that such complex values are not physically significant.

6. Numerical results and discussion. An approximate expression for the characteristic equation (5.11) is obtained if we expand the hyperbolic tangents in power series to the third power in $\gamma$. We find

$$
\frac{B_{1}}{B} \varphi_{1}=\zeta \gamma(1+\alpha)-\frac{1}{3} \gamma^{3}[2-(1-\alpha) \zeta] .
$$

If the wave length of the folding is sufficiently large, $\gamma$ and $\zeta$ are small. We may further neglect $(1-\alpha) \zeta$ in the bracket. Also we may assume $\zeta_{1}$ to be small, which amounts to neglecting the prestress $P_{1}$ in the surrounding medium. Hence we may put $\varphi_{1}=1$. Equation (6.1) then reduces to

$$
\frac{B_{1}}{B}=\zeta \gamma(1+\alpha)-\frac{2}{3} \gamma^{3} .
$$

This may also be written

$$
P=\frac{1}{12} B(l h)^{2}+\frac{B_{1}}{l h} .
$$

This last equation coincides with Eq. (3.1) of [1] obtained by neglecting the prestress $P_{1}$ in the surrounding medium and using a "thin plate" type theory for the layer. A complete discussion of the approximate equation (6.3) was given in [1] for the general case of two media of arbitrary viscoelastic properties and for a certain number of particular cases.

The exact equation (5.11) provides a means to test the accuracy of the approximate theory. For this purpose it is sufficient to compare the two theories on a simple example. We shall choose the case of two incompressible fluids. Incompressible fluids are represented by

$$
\begin{aligned}
\bar{R} & =\bar{R}_{1}=\infty, \\
\alpha & =\alpha_{1}=0, \\
\bar{Q} & =\mu p, \\
\bar{Q}_{1} & =\mu_{1} p,
\end{aligned}
$$

where $\mu$ is the viscosity coefficient of the layer and $\mu_{1}$ that of the surrounding medium. Introducing these expressions into the exact characteristic equation (5.11), we derive

$$
\frac{\mu_{1}}{\mu} \varphi_{1}=\frac{1}{\zeta} \tanh \gamma-\frac{(1+\zeta)^{2}}{\zeta} k \tanh k \gamma,
$$

where

$$
\begin{gathered}
\varphi_{1}=\frac{2-2 \zeta_{1}^{2}-\zeta_{1}^{3}}{k_{1}\left(1+\zeta_{1}\right)^{2}+1} \\
\zeta=\frac{P}{2 \mu p}, \quad \zeta_{1}=\frac{P_{1}}{2 \mu_{1} p}
\end{gathered}
$$


There are two types of approximations involved in Eq. (6.2): that due to the use of thin plate theory and the other due to neglecting the prestress $P_{1}$ of the surrounding medium. Let us discuss the effect of each separately. Therefore we put $\varphi_{1}=1$ in Eq. (6.5), i.e., we consider first the error due to the use of plate theory alone. The characteristic equation is then

$$
\frac{\mu_{1}}{\mu}=\frac{1}{\zeta} \tanh \gamma-\frac{(1+\zeta)^{2}}{\zeta} k \tanh k \gamma .
$$

This is a relation between these variables representing a function $\zeta$ of $\gamma$ with a parameter $\mu / \mu_{1}$. The variable $\zeta$ versus $\gamma$ is plotted in Figs. (4) and (5) for six values of $\mu / \mu_{1}$.

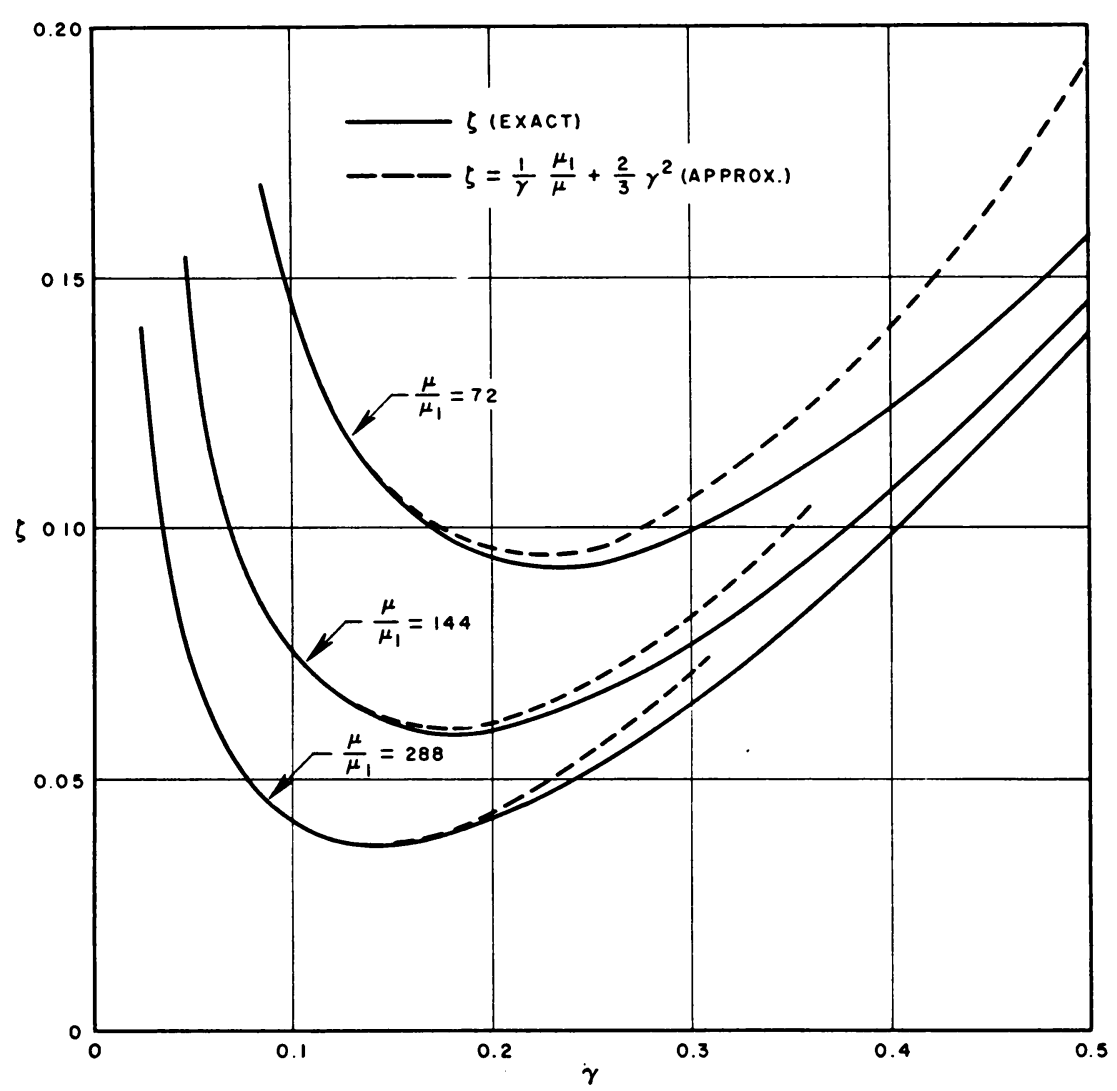

FIG. 4. Plot of $\zeta=P / 2 \mu p$ versus $\gamma$ and the parameter $\mu / \mu_{1}$ from the exact relation (6.7) and the approximate solution (6.9).

$$
\mu / \mu_{1}=6,12,36,72,144,288 .
$$

Consider now the approximate equation (6.2) which corresponds to the thin plate theory and, also, to neglect of the prestress $P_{1}$. For incompressible fluids, this equation becomes

$$
\frac{\mu_{1}}{\mu}=\zeta \gamma-\frac{2}{3} \gamma^{3}
$$


or

$$
\zeta=\frac{1}{\gamma} \frac{\mu_{1}}{\mu}+\frac{2}{3} \gamma^{2} .
$$

This also represents a family of functions $\zeta$ of $\gamma$ which are plotted as dotted lines in Figs. (4) and (5) for the same values of $\mu / \mu_{1}$. Examination of the two plots affords an immediate comparison between the exact and approximate theories. Of particular interest is the minimum value of $\zeta$ denoted by $\zeta_{\min }$ and the value $\gamma$ at which this minimum occurs. It is easily seen that the minimum of $\zeta$ for a given value of the compressive load $P$

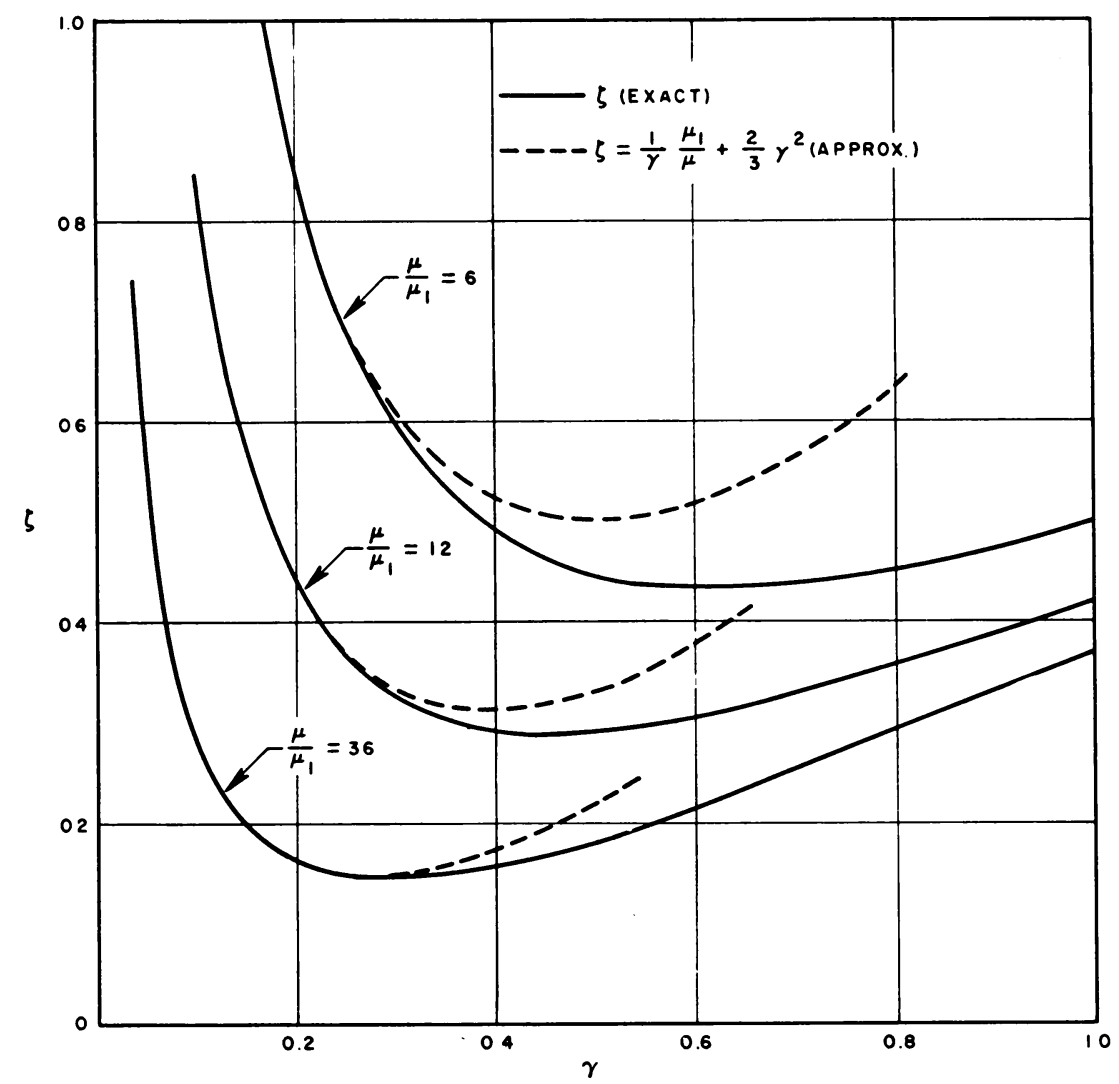

FIG. 5. Same as Fig. 4.

corresponds to maximum values of $p$. The significance of a root $p$ of Eq. (6.7) is given by the fact that for such roots a disturbance of wave number $l$ of the layer increases proportionally to the factor $e^{p t}$. Hence, maximum values of $p$ correspond to wave lengths of maximum rate of growth. We have referred to this wave length as the dominant wave length. The wave number $l_{d}$ of this dominant wave length is therefore determined by

$$
\gamma_{d}=\frac{1}{2} l_{d} h=\pi \frac{h}{L_{d}}
$$

( $L_{d}=$ dominant wave length). 
From formula (6.9) we find the approximate values

$$
\begin{aligned}
\gamma_{d} & =\left(\frac{3}{4} \frac{\mu_{1}}{\mu}\right)^{1 / 3}, \\
\zeta_{\min } & =2\left(\frac{3}{4} \frac{\mu_{1}}{\mu}\right)^{2 / 3} .
\end{aligned}
$$

The approximate value (6.11) of $\gamma_{d}$ as a function of $\left(\mu_{1} / \mu\right)^{1 / 3}$ is shown in Fig. 6 by the dotted straight line, while the exact value of $\gamma_{d}$ [determined from Eq. (6.7)] is represented by the full line. Comparison between the two curves indicates that a discrepancy in the value of $\gamma_{d}$ begins to be noticeable for values $\left(\mu_{1} / \mu\right)^{1 / 3}>1 / 4$ or $\mu / \mu_{1}<64$. The error in the dominant wave length may rise to $10-15 \%$ when $\mu / \mu_{1}$ becomes of the order of ten.

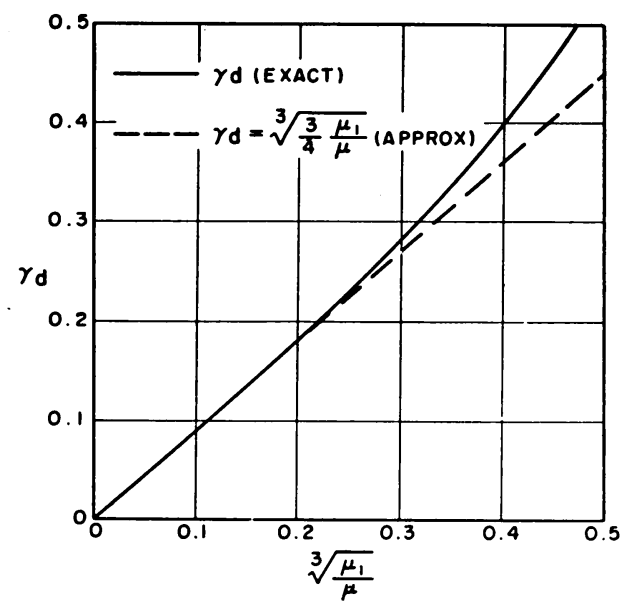

FIG. 6. Value $\gamma_{d}=\pi h / L_{d}\left(L_{d}=\downarrow, \mathrm{m}_{1} \quad\right.$ it wavelength $)$ as a function of $\mu / \mu_{1}$ by exact and approximate theory.

We have also plotted the exact value of $\zeta_{\min }$ versus $\left(\mu_{1} / \mu\right)^{2 / 3}$ as determined from Eq. (6.7). It is represented by the full line in Fig. 7. The approximate value (6.12) is represented by a dotted straight line. The error becomes noticeable for $\left(\mu_{1} / \mu\right)^{2 / 3}>1 / 16$; hence, again for $\mu / \mu_{1}<64$ For smaller values of $\mu / \mu_{1}$, the error may reach $10-15 \%$.

Let us now look upon the other approximation involved in Eq. (6.9) and which is due to neglect of the prestress $P_{1}$ in the surrounding medium. In order to evaluate the effect of this prestress, we must reintroduce the factor $\varphi_{1}$ which we had put equal to unity. This amounts to using the same graphs as in Figs. 4 to 7 , but with a corrected value $\mu_{1} \varphi_{1}$ instead of $\mu_{1}$ for the viscosity of the surrounding medium. Let us evaluate the value of the correction factor $\varphi_{1}$ in the vicinity of the dominant wave number (near $\left.\zeta_{\min }\right)$. Assuming the layered material to be squeezed uniformly so that the strains are the same in the layer and the surrounding medium the compressive prestresses $P_{1}$ and $P$ satisfy relation (5.1) and we derive

$$
\zeta=\zeta_{1}
$$

We may, therefore, evaluate $\varphi_{1}$ as a function of $\zeta$. Values of $\varphi_{1}$ are given in Table 3 . 
TABLE 3.

\begin{tabular}{c|c|c|c}
\hline \hline$\zeta$ & $\varphi_{1}$ & $\left(\varphi_{1}\right)^{\frac{3}{3}}$ & $\varphi_{\mathrm{i}}{ }^{2 / 3}$ \\
\hline .1 & .946 & .980 & .962 \\
.3 & .804 & .930 & .865 \\
.5 & .598 & .841 & .710 \\
\hline
\end{tabular}

The values of $\varphi_{1}$ show a decrease in the "effective value" $\mu_{1} \varphi_{1}$ of the viscosity coefficient of the surrounding medium due to the prestress. The decrease is about $5 \%$ or less for values of $\zeta_{\min }<0.1$, i.e., for $\mu / \mu_{1}>64$ and may be as high as $40 \%$ for the lower values of $\mu / \mu_{1}$. The effect on the dominant wave length is much smaller and is proportional to $\left(\varphi_{1}\right)^{1 / 3}$. From the values of $\left(\varphi_{1}\right)^{1 / 3}$ in Table 3 and from Fig. 6, it can be seen that the dominant wave length will be increased from 2 to 16 per cent in the same range. Reference to Fig. 7 and values of $\left(\varphi_{1}\right)^{2 / 3}$ show that $\zeta_{\min }$ will be decreased by an amount somewhat larger

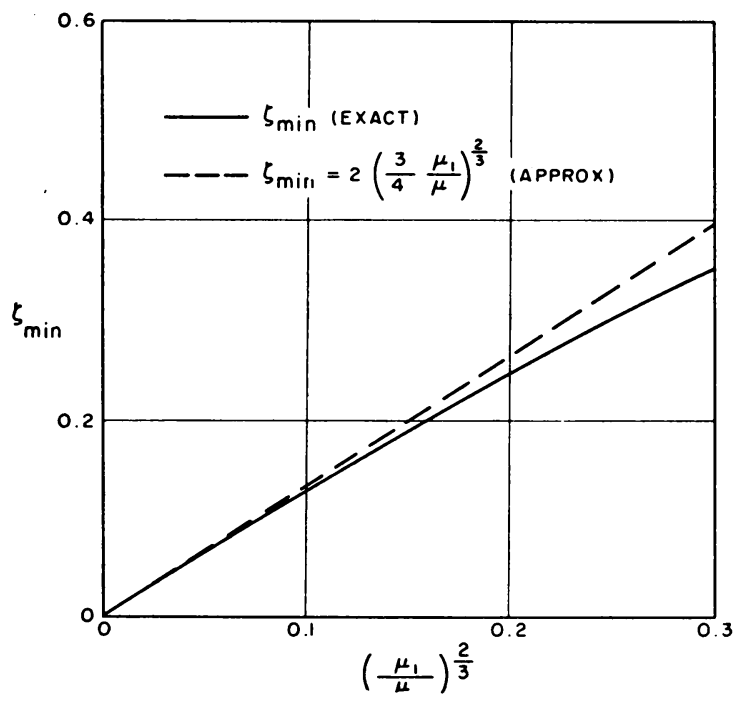

Fig. 7. The minimum value of $\zeta$ as a function of $\mu / \mu_{1}$ by exact and approximate theory.

due to the prestress. In general, we may conclude that the effect of the prestress $P_{1}$ in the embedding medium becomes negligible if the viscosity ratio $\mu / \mu_{1}$ of the layer to the medium is larger than about 64 . It is interesting to note that this was also found to be the condition for the error, due to thin plate theory, to be negligible.

In order to complete the present discussion, we must also pay attention to the degree of instability involved in the phenomenon of folding. The dominant wave length determined by $\gamma_{d}$ is that for which an initial waviness in the layer increases at the fastest rate. After a time $t$, the amplitude is multiplied by the factor $e^{p t}$ where $p$ is given by

$$
p=\frac{P}{2 \mu \zeta_{\min }} .
$$

We may ask, for instance, what is the value of the factor $e^{p t}$ after a time $t_{1}$ such that 
the layered material is squeezed by 25 per cent under the compressive stresses $P$ and $P_{1}$.

Referring to the same argument as in Sec. 4 in the case of the half space, we find

$$
t_{1}=\frac{\mu}{P}
$$

and

$$
p t_{1}=1 / 2 \zeta_{\min } .
$$

From Fig. 7 giving $\zeta_{\min }$ as a function of $\mu / \mu_{1}$, we may derive the amplification factor $e^{p t_{1}}$ in terms of $\mu / \mu_{1}$. Values are shown in Table 4.

TABLE 4.

\begin{tabular}{r|c}
\hline \hline$/ \mu_{1}$ & $\exp \left(p t_{1}\right)$ \\
\hline 8 & 3.78 \\
27 & 14.8 \\
64 & 127 \\
125 & 1940 \\
& \\
\hline
\end{tabular}

The amplification factor increases sharply and becomes quite large for values $\mu / \mu_{1}>64$. Below these values the magnitude of the amplification factor becomes relatively small, indicating that the instability in that range is not too significant and will not exhibit very sharp features. This lower range of values is also the one in which the errors become appreciable if we apply thin plate theory and neglect the prestress in the surrounding medium. We may conclude that in the region of significant instability Eq. (6.2) of the approximate theory is applicable.

The present discussion is limited to the case of incompressible viscous fluids. A similar discussion can be carried out for any two materials of arbitrary viscoelastic properties represented by four suitable operators. If the two materials are incompressible, the plots in Figs. 4 to 7 represent master graphs by which the folding may be analyzed quantitatively by means of very simple calculations.

\section{REFERENCES}

1. M. A. Biot, Folding instability of a layered viscoelastic medium under compression, Proc. Roy. Soc, A 242, 444-445 (1957)

2. M. A. Biot, On the instability and folding deformation of a layered viscoelsatic medium in compression J. Appl. Mech. (in press)

3. M. A. Biot, Theory of elasticity with large displacements and rotations, Proc. Fifth Intern. Congr. Appl. Mechanics, 1938

4. M. A. Biot, Non-linear theory of elasticity and the linearized case for a body under initial stress, Phil. Mag., Sec. 7, XXVII, 468-489 (April 1939)

5. M. A. Biot, Elastizitats Theorie zweiter Ordnung mit Anwendungen, Z. Angew. Math. u. Mech. 20, No. 2, 89-99 (April 1940)

6. M. A. Biot, The influence of initial stress on elastic waves, J. Appl. Phys. 11, No. 8, 522-530 (August 1940)

7. M. A. Biot, Theory of stress-strain relations in anisotropic viscoelasticity and relaxation phenomena, J. Appl. Phys. 25, No. 11, 1385-1391 (November 1954) 